\title{
COMMENTARY
}

\section{Acute kidney injury in sepsis: transient or intrinsic?}

\author{
Achim Jörres \\ See related research by Vanmassenhove et al., http://ccforum.com/content/17/5/R234
}

\begin{abstract}
The negative prediction of intrinsic versus transient acute kidney injury (AKI) in septic patients may be facilitated by combined assessment of fractional excretion of sodium and urea. If both excretions are high this would signal the presence of transient AKI and suggest that successful restoration of diuresis by conservative therapy is likely, thus supporting a waitand-watch approach regarding the initiation of acute renal replacement therapy.
\end{abstract}

Acute kidney injury (AKI) is a common problem in the ICU, particularly in patients with severe sepsis and septic shock [1]. Opposite to earlier belief, AKI is not necessarily a fully reversible complication of critical illness but may result in persistent kidney damage, progressive renal disease, or even excess morbidity and mortality in the long run [2]. Identifying patients at risk, detecting early AKI as soon as possible, and initiating appropriate therapy without delay are thus essential. To this end, a considerable number of novel biomarkers for AKI were developed during recent years and tested in clinical trials; however, very little of value for earlier prognostication has transpired to date. Specifically, it often remains a difficult task to differentiate between transient, reversible AKI and intrinsic AKI (that is, structural kidney damage that is not rapidly reversible following restoration of systemic and renal blood flow by appropriate early goal-directed therapy). In clinical practice, therefore, fluid responsiveness is often tested following the principle of trial and error, with potential adverse consequences if the fluid challenge does not lead to improved kidney function but rather results in overhydration [3].

Correspondence: achim.joerres@charite.de

Department of Nephrology and Medical Intensive Care, Charité University Hospital Campus Virchow-Klinikum, Augustenburger Platz 1, Berlin 13353, Germany
In their present study, Vanmassenhove and colleagues attempt to define the value of traditional (fractional excretion of sodium and urea) and novel (neutrophil gelatinase-associated lipocalin) markers for AKI in the prognostication of septic AKI; that is, in differentiating transient (pre-renal) from intrinsic renal failure [4]. Their data support a combination of fractional excretion of sodium and urea to allow the negative prediction of intrinsic AKI with high predictive value. Such negative prediction would be of potential clinical relevance because in these patients the initiation of acute renal replacement therapy might be deferred and the effect of conservative therapy observed. On the other hand, in cases where intrinsic AKI seems probable and further progression to kidney failure must be expected, early initiation of acute renal replacement therapy well before overt complications such as hyperkalemia, severe metabolic acidosis, or overhydration occur would seem prudent.

Moreover, the data from the present study support the view that an underdiagnosis of AKI may result if the diuresis criterion of classification systems is omitted. However, one has to keep in mind that therapeutic measures may interfere with the diagnostic value of urine output, fractional sodium excretion and also serum creatinine. Administration of loop diuretics may or may not lead to enhanced diuresis but also to increased sodium excretion, while fluid resuscitation may result in dilution of serum creatinine particularly in the anuric patient. Furthermore, the type of fluid used for resuscitation may be relevant, as can be derived from the recent Crystalloid versus Hydroxyethyl Starch Trials study [5]. Here, posthoc analysis of Risk, Injury, Failure, Loss of function, End-stage kidney disease (RIFLE) categories favored hydroxyethyl starch when the urine output criterion was used, but favored saline when the creatinine criterion was used - suggesting that fluid resuscitation with hydroxyethyl starch may initially (transiently) conserve 
urine output but at the same time lead to a reduced glomerular filtration rate.

Whilst the present study clearly reminds us of the virtues and values of traditional knowledge even in modern times when simple tests and procedures are replaced by novel, more sophisticated (and typically more expensive) methods, the search for biomarkers allowing a better prognostication of AKI must continue. In addition to neutrophil gelatinase-associated lipocalin, insulin-like growth factor binding protein-7 was recently proposed as a promising candidate [6] that will have to be tested in adequately powered prospective studies. For the time being, however, the 'renal troponin' remains an unfulfilled desire, with the intensivist's clinical experience and expertise constituting the most important asset to guide decisions.

\section{Abbreviations}

AKI: Acute kidney injury; RIFLE: Risk injury, failure, loss of function, end-stage kidney disease.

\section{Competing interests}

The author declares that he has no competing interests.

\section{Published: 20 Nov 2013}

\section{References}

1. Oppert M, Engel C, Brunkhorst FM, Bogatsch H, Reinhart K, Frei U, Eckardt KU, Loeffler M, John S, German competence network sepsis (sepnet): Acute renal failure in patients with severe sepsis and septic shock - a significant independent risk factor for mortality: results from the german prevalence study. Nephrol Dial Transplant 2008, 23:904-909.

2. Coca SG, Singanamala S, Parikh CR: Chronic kidney disease after acute kidney injury: a systematic review and meta-analysis. Kidney Int 2012, 81:442-448

3. Bouchard J, Soroko SB, Chertow GM, Himmelfarb J, Ikizler TA, Paganini EP, Mehta RL, Program to improve care in acute renal disease (PICARD) study group: Fluid accumulation, survival and recovery of kidney function in critically ill patients with acute kidney injury. Kidney Int 2009, 76:422-427.

4. Vanmassenhove J, Glorieux G, Hoste E, Dhondt A, Van Biesen W: Urinary output and fractional excretion of sodium and urea as indicators of transient versus intrinsic acute kidney injury during early sepsis. Crit Care 2013, 17:R234

5. Myburgh JA, Finfer S, Bellomo R, Billot L, Cass A, Gattas D, Glass P, Lipman J, Liu B, McArthur C, McGuinness S, Rajbhandari D, Taylor CB, Webb SA, CHEST investigators; Australian and New Zealand intensive care society clinical trials group: Hydroxyethyl starch or saline for fluid resuscitation in intensive care. N Engl J Med 2012, 367:1901-1911.

6. Aregger F, Uehlinger DE, Witowski J, Brunisholz RA, Hunziker P, Frey FJ Jörres A: Identification of IGFBP-7 by urinary proteomics as a novel prognostic marker in early acute kidney injury. Kidney Int 2013 [Epub ahead of print].

$10.1186 / \mathrm{cc} 13124$

Cite this article as: Jörres: Acute kidney injury in sepsis: transient or intrinsic? Critical Care 2013, 17:1014 